\title{
Correction to: Predicting drug response of tumors from integrated genomic profiles by deep neural networks
}

\author{
Yu-Chiao Chiu', Hung-l Harry Chen 1,2, Tinghe Zhang ${ }^{2}$, Songyao Zhang ${ }^{2,3}$, Aparna Gorthi', Li-Ju Wang',
} Yufei Huang ${ }^{2,4^{*}}$ and Yidong Chen ${ }^{1,4^{*}}$

\section{Correction to: BMC Medical Genomics 2019, 12(Suppl 1):18. https://doi.org/10.1186/s12920-018-0460-9}

Following publication of the original article [1], the authors provided an updated funding statement to the article. The updated statement is as follows:

\section{Funding}

This research and this article's publication costs were supported partially by the NCI Cancer Center Shared Resources (NIH-NCI P30CA54174 to YC), NIH (CTSA 1UL1RR025767-01 to YC, and R01GM113245 to YH), CPRIT (RP160732 to YC), and San Antonio Life Science Institute (SALSI Innovation Challenge Award 2016 to $\mathrm{YH}$ and YC and SALSI Postdoctoral Research Fellowship to YCC). AG is supported by the NIH/NCATS TL1 Translational Science Training award (TL1TR002647) and the AACR-AstraZeneca Stimulating Therapeutic Advances through Research Training grant. The funding sources had no role in the design of the study and collection, analysis, and interpretation of data and in writing the manuscript.

\footnotetext{
Author details

'Greehey Children's Cancer Research Institute, University of Texas Health Science Center at San Antonio, San Antonio, TX 78229, USA. ${ }^{2}$ Department of Electrical and Computer Engineering, The University of Texas at San Antonio, San Antonio, TX 78249, USA. ${ }^{3}$ Laboratory of Information Fusion Technology of Ministry of Education, School of Automation, Northwestern Polytechnical University, Xi'an 710072, Shaanxi, China. ${ }^{4}$ Department of Epidemiology and Biostatistics, University of Texas Health Science Center at San Antonio, San Antonio, TX 78229, USA.
}

Published online: 12 August 2019

\section{Reference}

1. Chiu et al. BMC Medical Genomics 2019, 12(Suppl 1):18. Predicting drug response of tumors from integrated genomic profiles by deep neural networks https://doi.org/10.1186/s12920-018-0460-9.

*Correspondence: Yufei.Huang@utsa.edu; ChenY8@uthscsa.edu

${ }^{2}$ Department of Electrical and Computer Engineering, The University of Texas at San Antonio, San Antonio, TX 78249, USA

'Greehey Children's Cancer Research Institute, University of Texas Health

Science Center at San Antonio, San Antonio, TX 78229, USA

Full list of author information is available at the end of the article

C The Author(s). 2019 Open Access This article is distributed under the terms of the Creative Commons Attribution 4.0 International License (http://creativecommons.org/licenses/by/4.0/), which permits unrestricted use, distribution, and reproduction in any medium, provided you give appropriate credit to the original author(s) and the source, provide a link to the Creative Commons license, and indicate if changes were made. The Creative Commons Public Domain Dedication waiver (http://creativecommons.org/publicdomain/zero/1.0/) applies to the data made available in this article, unless otherwise stated. 